\title{
Portal vein thrombosis after splenectomy for gastric malignant lymphoma
}

\author{
Kazumasa Fujitani, Akiko Nishiyama, Toshimasa Tsujinaka, Motohiro Hirao, Yasunori Hasuike, \\ and YutaKa TAKEDA \\ Department of Surgery, Osaka National Hospital, 2-1-14 Hoenzaka, Chuo-ku, Osaka 540-0006, Japan
}

\begin{abstract}
Portal vein thrombosis (PVT) has rarely been documented in patients after splenectomy for gastric malignancy. We report a case of PVT that occurred after splenectomy as part of an en-bloc node dissection performed to treat gastric malignant lymphoma. A 38-year-old man underwent total gastrectomy and splenectomy with en-bloc D2 lymph node dissection. The spleen weighed $480 \mathrm{~g}$. On postoperative day (POD) 31 , the patient complained of abdominal pain in the right upper quadrant accompanied by fever. Moderate elevations of C-reactive protein (CRP), aspartate transaminase (AST), and alanine transaminase (ALT) were noted. Contrast-enhanced computed tomography (CT) and ultrasonography disclosed thrombus in the portal vein and the splenic vein. There were no abnormalities in the levels of lupus anticoagulant, protein $C$ antigen, protein S antigen, or antithrombin III (AT III). A diagnosis of PVT was made, and prompt treatment, including intravenous heparin combined with tissue plasminogen activator (tPA) was initiated, followed by longterm warfarin. This treatment resulted in clinical improvement, but failed to achieve thrombolysis in the portal vein. At follow-up after 6 months, the patient complained of postprandial abdominal pain with persistent peripheral edema and ascites. This case indicates that splenectomy for en-bloc node dissection in gastric malignancy is a possible cause of PVT. Because both the symptoms and the laboratory data in PVT are nonspecific, a high level of clinical suspicion and a low threshold for obtaining imaging examinations are important in the early diagnosis of PVT. Surgeons should remember PVT among several other complications whenever patients treated with radical gastrectomies are symptomatic and imaging studies are considered necessary.
\end{abstract}

Key words Portal vein thrombosis - Splenectomy - Gastric malignant lymphoma

Offprint requests to: $\mathrm{K}$. Fujitani

Received: April 18, 2003 / Accepted: July 8, 2003

\section{Introduction}

Portal vein thrombosis (PVT) is a rare disorder in adults and is usually associated with cirrhosis; abdominal infections, such as pancreatitis and peritonitis; or neoplastic diseases, such as pancreatic cancer and hepatocellular carcinoma [1]. PVT also develops postoperatively, mainly after splenectomy performed for hematological disorders [2]. Splenectomy is also frequently carried out in combination with total gastrectomy to achieve complete lymph node dissection in patients with gastric malignancy, although PVT has rarely been documented in such patients. We report a case of PVT that occurred after splenectomy performed as part of an en-bloc node dissection to treat gastric malignant lymphoma.

\section{Case report}

A 38-year-old man was referred to our department for surgical treatment of malignant lymphoma located in both the upper and the lower third of the stomach. On physical examination, the liver and spleen were palpable, with no peripheral lymphadenopathy. Except for a decreased total protein level $(4.6 \mathrm{~g} / \mathrm{dl})$ and anemia (hemoglobin, $10.5 \mathrm{~g} / \mathrm{dl}$ ), the hematological and biochemical findings were normal, including bleeding time $(2.0 \mathrm{~min})$, prothrombin time $(11.9 \mathrm{~s})$, activated partial thrombin time $(37.7 \mathrm{~s})$, plasma fibrinogen level $(482 \mathrm{mg} / \mathrm{dl})$, and platelet count $\left(173 \times 10^{9} / 1\right)$. He had not received any preoperative blood transfusions. Qualitative platelet function was not tested. Preoperative computed tomography $(\mathrm{CT})$ revealed thickening of the stomach wall, but no evidence of metastasis, including in the regional lymph nodes close to the stomach. A patent portal vein and enlarged spleen and liver were also seen (Fig. 1A).

He underwent total gastrectomy, splenectomy, and cholecystectomy with en-bloc D2 lymph node dissec- 

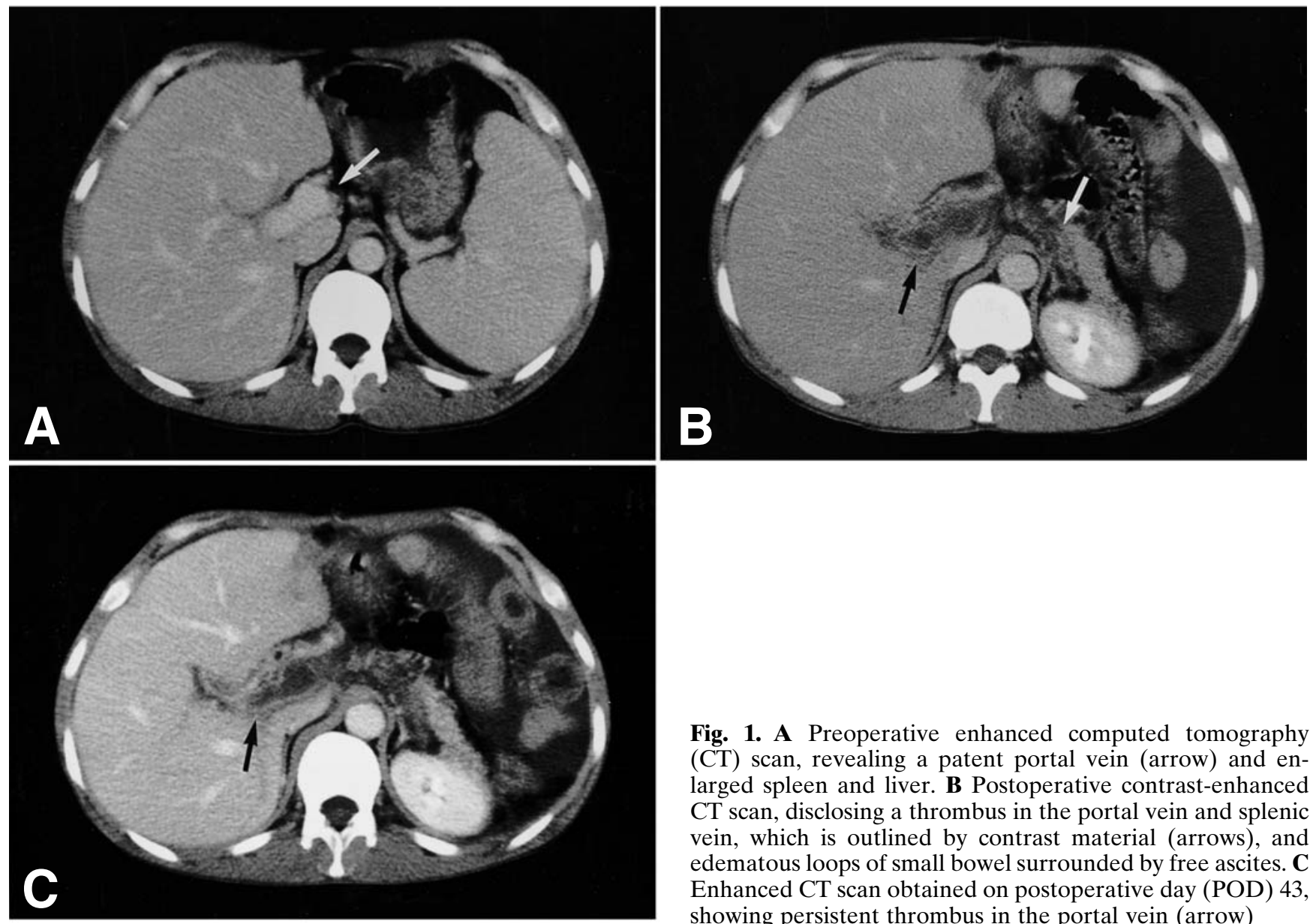

Fig. 1. A Preoperative enhanced computed tomography (CT) scan, revealing a patent portal vein (arrow) and enlarged spleen and liver. B Postoperative contrast-enhanced CT scan, disclosing a thrombus in the portal vein and splenic vein, which is outlined by contrast material (arrows), and edematous loops of small bowel surrounded by free ascites. $\mathbf{C}$ Enhanced CT scan obtained on postoperative day (POD) 43, showing persistent thrombus in the portal vein (arrow)

tion, according to the the Japanese Classification of Gastric Carcinoma [3]. Splenectomy was performed to achieve an en-bloc node dissection. The splenic vein was doubly ligated close to the pancreatic tail, and the spleen weighed $480 \mathrm{~g}$. The operation time was $302 \mathrm{~min}$. Blood loss was $1500 \mathrm{ml}$, and 4 units of packed red cells were transfused. Histological diagnosis of the primary gastric lesion, according to the REAL classification, was non-Hodgkin diffuse large B-cell lymphoma, and lymphoma cell infiltration of the serosa was observed, as shown in Fig. 2B. Immunohistochemical studies of the resected gastric specimen revealed that the lymphoma cells were positive for CD45, CD20, and CD79a, and negative for CD3 and CD30. Microscopic examination of the regional lymph nodes and spleen revealed no evidence of an involvement by the lymphoma cells. Clinical stage was IE according to the Ann Arbor classification of lymphoma [4]. Postoperative recovery was uneventful, and the patient was discharged on postoperative day (POD) 26.

On POD 31, he was readmitted, complaining of severe abdominal pain in the right upper quadrant, accompanied by fever. Moderate elevations of C-reactive protein $(10.2 \mathrm{mg} / \mathrm{dl})$, aspartate transaminase (AST;
123 IU/l), and alanine transaminase (ALT; 100IU/l) values were noted, without any leukocytosis or thrombocytosis. The bilirubin level was not elevated. Contrast-enhanced CT (Fig. 1B) and ultrasonography on POD 32 disclosed thrombus in the portal vein and splenic vein. Ascites and pleural effusion were also noted, without any evidence of recurrence of the disease. The intestine appeared to be edematous and surrounded by free ascites. After the diagnosis of PVT, prompt treatment, including intravenous heparin combined with human recombinant tissue plasminogen activator (tPA) was initiated. The tPA $\left(6.40 \times 10^{6} \mathrm{U} /\right.$ day) was given for 2 days, and heparin (20000 U/day) was given for 10 days, together with antibiotics. Mildly elevated D-dimer $(2.63 \mu \mathrm{g} / \mathrm{ml})$ and thrombinantithrombin III complex (TAT; $5.1 \mathrm{ng} / \mathrm{ml}$ ) levels were noted prior to the start of heparin therapy. There were no abnormalities in the levels of lupus anticoagulant (1.27), protein $\mathrm{C}$ antigen (79\%; normal range [NR], $64 \%-146 \%$ ), protein S antigen (97\%; NR, 65\%-135\%), or antithrombin III (119\%; NR, 75\%-125\%). By POD 43 , this treatment had resulted in clinical improvement, but had failed to achieve thrombolysis in the portal vein (Fig. 1C). Systemic heparinization was gradually re- 

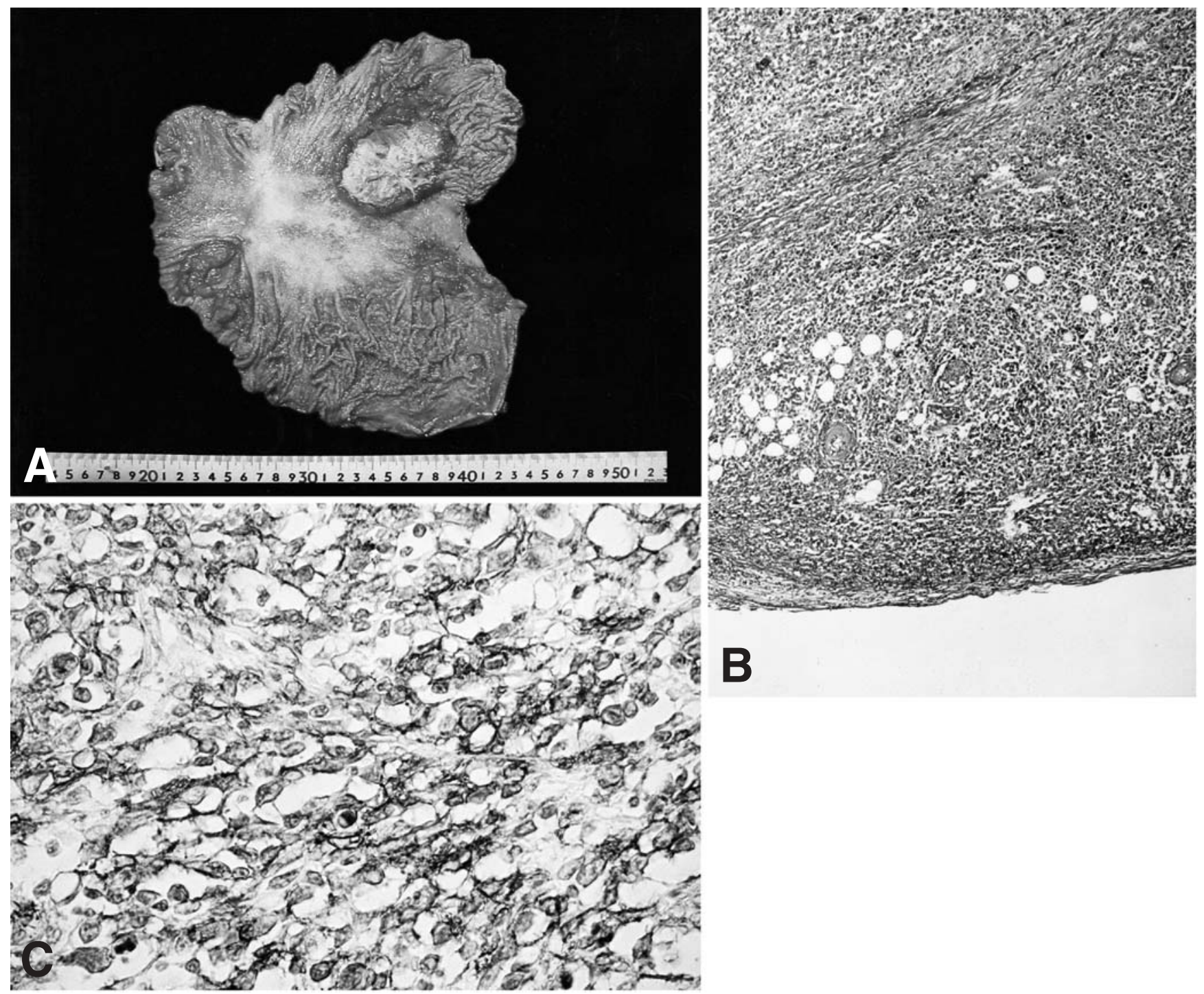

Fig. 2A-C. Pathological findings in the resected stomach. A Gross appearance of the stomach, showing two lymphoma lesions in the upper third and one lesion in the lower third of the stomach. B Low-power view of the gastric tumor, showing diffuse-type lymphoma infiltrating the serosa. $\mathbf{C ~ C D} 20$ immunostaining, revealing positive reactivity in neoplastic cells. $\mathbf{B} \mathrm{H} \& \mathrm{E}, \times 16 ; \mathbf{C} \times 120$

placed by antithrombotic therapy with daily sodium warfarin monotherapy, and the patient was discharged on POD 57.

The patient has been followed for more than 6 months without adjuvant chemotherapy or any evidence of recurrence of the disease. At follow-up after 6 months, the patient complained of postprandial abdominal pain with persistent peripheral edema and ascites.

\section{Discussion}

PVT after splenectomy occurs most commonly in patients with hematological disorders, who frequently have moderate to marked splenomegaly with a dilated splenic vein and are in a hypercoagulable state [5]. Chronic hematologic diseases, such as myeloproliferative disorders and hemolytic anemia, are recognized as significant risk factors for PVT after splenectomy [5-7]. In contrast, PVT has rarely been reported after the removal of a normal spleen from patients without hematological disorders [2]. So far, there have been reports of only two patients who developed PVT after splenectomy for en-bloc node dissection in gastric malignancy $[2,8]$, excluding our patient.

Stasis of blood flow in the stump of the splenic vein is considered to be a pathogenic cause of PVT after splenectomy. In patients with a large spleen, the stump of the splenic vein remaining after ligation forms a cul de 
sac $[2,6,9,10]$. Turbulence in this cul de sac can result in thrombosis, which, in turn, may extend into the portal vein and the superior mesenteric vein. Because there is a correlation between the size of the spleen and the diameter of the splenic vein, marked splenomegaly with large veins increases the risk of PVT $[2,6,9]$. Some authors believe that a long splenic vein stump increases the risk for PVT as well, and they recommend ligation of the splenic vein as close as possible to its junction with the inferior mesenteric vein so that there is some continuous flow in the remaining section of the splenic vein $[2,6,11,12]$. In addition, the increased blood viscosity, due to high platelet and leukocyte counts as a result of lack of splenic breakdown, is considered to cause hypercoagulability after splenectomy $[2,11]$. The median platelet count at the time of PVT diagnosis has been reported to be $827 \times 10^{9} / 1$ (range, $375-1447 \times 10^{9} /$ 1), while the median postoperative maximum platelet count has been reported to be $1020 \times 10^{9} / 1$ (range, 609$1490 \times 10^{9} / 1$ [7]. The maximal platelet count in our patient was $719 \times 10^{9} / 1$ on POD 12, and the high platelet counts may have caused hypercoagulability after the splenectomy. In addition, the median splenic weight in patients who developed PVT was documented to be $660 \mathrm{~g}$ (mean, 975 g; range, 240-2730g) [7]. Our patient's spleen weighed $480 \mathrm{~g}$, which suggested a large splenic vein stump that led to sluggish blood flow after the splenectomy.

The incidence of symptomatic PVT after splenectomy has ranged from $0.7 \%$ to $1.6 \%$ in large retrospective studies $[2,7,11,13]$, while that of asymptomatic PVT has been reported to be $3.8 \%-6.7 \%[11,14]$. The symptoms of PVT include abdominal pain, fever, diarrhea, nausea, ascites, and peripheral edema $[2,6,14,15]$. These symptoms are nonspecific, which makes it difficult to suspect PVT initially in the majority of patients $[2,6,14]$. The laboratory data are also considered to be nonspecific and unhelpful in establishing the diagnosis $[1,2]$. However, leukocytosis and mild elevations in transaminases, alkaline phosphatase, lactate dehydrogenase, and bilirubin have been reported so far [1,2], and they can be clues to suspect PVT. On the other hand, contrastenhanced CT and ultrasonography can easily detect PVT, as in our patient. Therefore, suspicion of PVT is important for the early diagnosis of PVT.

PVT is a potentially fatal complication of splenectomy. The major complication of such thrombosis is extension of the clot into the superior mesenteric vein, with resultant lethal bowel infarction $[1,2,6]$. Therefore, prompt initiation of thrombolytic therapy for PVT is important for successful management [7]. Treatment with thrombolytic agents followed by longterm anticoagulants, in combination with antibiotics, can prevent the development of lethal complications such as bowel infarction and variceal bleeding [2]. A high thromboly- sis rate, of $77 \%$, has been demonstrated after anticoagulant therapy [14], and fibrinolytic therapy with highdose urokinase $\left(2.16 \times 10^{6} \mathrm{U}\right)$ over 3 days has been documented to dissolve PVT successfully [12]. However, optimal dosages and a standard method of thrombolytic therapy for PVT after splenectomy have not been clearly established. The role and optimal duration of anticoagulant therapy are also unclear. Some authors prescribe longterm anticoagulants only for symptomatic patients [2], whereas others prescribe them for all patients with proven PVT [14]. The role of prophylactic heparin remains uncertain as well, because PVT sometimes develops even after the prophylactic use of heparin [7]. When a thrombus has persisted for months, cavernous transformation of the portal system may develop [2,14]. Once cavernous transformation occurs, the recommended duration of anticoagulant therapy is, as yet, unclear. In addition to medical therapy, more aggressive treatment, with a combination of surgical thrombectomy and regional thrombolysis using tPA has been proposed [16], and such procedures may improve the outcome in selected patients, such as those with signs of bowel infarction. In our patient, prompt therapy, including intravenous heparin combined with tPA, was initiated after the diagnosis of PVT, followed by longterm warfarin. This treatment succeeded in clinical improvement and prevented the lethal complications, but failed to achieve thrombolysis in the portal vein. It has been reported that treatment within 10 days after splenectomy is successful in achieving thrombolysis, but that delayed treatment is ineffective [7]. The initiation of treatment on POD 32 might have been too late in our patient. As regards the dosage of tPA and duration of anticoagulant therapy, administration of tPA, $6.40 \times 10^{6} \mathrm{U} /$ day for 2 days, and heparin, $20 \times$ $10^{3} \mathrm{U} /$ day for 10 days, appeared to be sufficient.

The diagnosis of PVT requires definitive imaging evidence. Ultrasonography is relatively inexpensive and easy to perform, and color-flow Doppler techniques supplement the accuracy of the examination and are currently considered to be the best method of diagnosing PVT. Contrast-enhanced CT is highly specific, but slightly less sensitive than ultrasonography [17]. Magnetic resonance imaging angiography and mesenteric angiography are unlikely to be necessary to establish the diagnosis in most cases. Color Doppler imaging has allowed symptomatic PVT to be diagnosed within 15 days after splenectomy, and asymptomatic PVT within 30 days $[9,11,14]$; thus, our patient should have been examined by ultrasonography within the first 30 days after surgery and may have benefited from early detection. There have been contradictory discussions regarding routine surveillance imaging for PVT after splenectomy $[14,15]$; however, such surveillance may lead to the early detection and treatment of PVT and 
thus, potentially, avoid subsequent complications. Because the natural history of PVT is unknown, only a randomized study can provide the answer as to whether the early detection and early treatment of asymptomatic PVT are of benefit to patients. However, a high level of clinical suspicion and a low threshold for obtaining imaging examinations is needed in symptomatic patients.

\section{Conclusion}

Although PVT after splenectomy has been reported mainly in patients with hematological disorders, the findings in our patient suggest that splenectomy for enbloc node dissection in gastric malignancy is a possible cause of PVT. Surgeons should remember PVT among several other complications whenever patients treated with radical gastrectomies are symptomatic and imaging studies are considered necessary.

\section{References}

1. Cohen J, Edelman RR, Chopra S. Portal vein thrombosis: a review. Am J Med 1992;92:173-82.

2. Rattner DW, Ellman L, Warshaw AL. Portal vein thrombosis after elective splenectomy. An underappreciated, potentially lethal syndrome. Arch Surg 1993;128:565-70.

3. Japanese Gastric Cancer Association: Japanese classification of gastric carcinoma, 2nd English edition. Gastric Cancer 1998;1:10 24.

4. Carbone P, Kaplan HS, Musshoff K, Smithers DW, Tubiana M. Report of the Committee on Hodgkin's Disease Staging Classification. Cancer Res 1971;31:1860-1.

5. Valla D, Casadevall N, Huisse MG, Tulliez M, Grange JD, Muller $\mathrm{O}$, et al. Etiology of portal vein thrombosis in adults. A prospec- tive evaluation of primary myeloproliferative disorders. Gastroenterology 1988;94:1063-9.

6. Broe PJ, Conley CL, Cameron JL. Thrombosis of the portal vein following splenectomy for myeloid metaplasia. Surg Gynecol Obstet 1981;152:488-92.

7. van't Riet M, Burger JWA, van Muiswinkel JM, Kazemier G, Schipperus MR, Bonjer H. Diagnosis and treatment of portal vein thrombosis following splenectomy. Br J Surg 2000;87:1229-33.

8. Kogire M, Nishida O, Kobayashi H, Shimogou T, Izumi F, Sugiyama A, et al. Splenectomy for en bloc node dissection in gastric cancer: a possible cause of portal vein thrombosis. Arch Jpn Chir 1996;65:49-53.

9. Eguchi A, Hashizume M, Kitano S, Tanoue K, Wada H, Sugimachi K. High rate of portal thrombosis after splenectomy in patients with esophageal varices and idiopathic portal hypertension. Arch Surg 1991;126:752-5.

10. Belli L, Romani F, Riolo F, Rondinara G, Aseni P, Di Stefano M, et al. Thrombosis of portal vein in absence of hepatic disease. Surg Gynecol Obstet 1989;169:46-9.

11. Chaffanjon PCJ, Brichon PY, Ranchoup Y, Gressin R, Sotto JJ. Portal vein thrombosis following splenectomy for hematologic disease: prospective study with Doppler color flow imaging. World J Surg 1998;22:1082-6.

12. Suzuki S, Nakamura S, Baba S, Sakaguchi S, Ohnuki Y, Yokoi Y, et al. Portal vein thrombosis after splenectomy successfully treated by an enormous dosage of fibrinolytic agent in a short period: report of two cases. Jpn J Surg 1992;22:464-9.

13. Kunin N, Desjardins JF, Letoquart JP, La Gamma A, Lebois E, Mambrini A. Portal system thrombosis after hematological splenectomy. J Chir (Paris) 1996;133:453-8.

14. Petit P, Bret PM, Atri M, Hreno A, Casola G, Gianfelice D. Splenic vein thrombosis after splenectomy: frequency and role of imaging. Radiology 1994;190:65-8.

15. Loring LA, Panicek DM, Karpeh MS. Portal system thrombosis after splenectomy for neoplasm or chronic hematologic disorder: is routine surveillance imaging necessary? J Comput Assist Tomogr 1998;22:856-60.

16. Demertzis S, Ringe B, Gulba D, Rosenthal H, Pichlmayr R. Treatment of portal vein thrombosis by thrombectomy and regional thrombolysis. Surgery 1994;115:389-93.

17. Subramanyam BR, Balthazar EJ, Lefleur RS, Horii SC, Hulnick DH. Portal venous thrombosis: correlative analysis of sonography, CT, and angiography. Am J Gastroenterol 1984;79:773-6. 